\section{Förderpreis „Neue immunologische Therapien atopischer/allergischer Erkrankungen"}

Zum ersten Mal vergibt die Deutsche Gesellschaft für Allergologie und klinische Immunologie (DGAKI) in diesem Jahr den Förderpreis "Neue immunologische Therapien atopischer/allergischer Erkrankungen". Gestiftet wird der neue Wissenschaftspreis von SanofiAventis Deutschland GmbH, Berlin. Der Preis steht im Kontext mit der neuen Arbeitsgruppe der DGAKI „Biologika in der Therapie atopischer Erkrankungen“, deren Gründungsveranstaltung ebenfalls für den Deutschen Allergiekongress angekündigt ist.

Der Preis dient der Auszeichnung besonderer wissenschaftlicher Arbeiten, die sich mit Fragestellungen zum Einsatz von neuen innovativen Therapieansätzen (z. B. spezifische Antikörper/ Biologika, Peptide, small molecules) bei atopischen beziehungsweise allergischen Erkrankungen befassen. 2017 werden $5.000 €$ zu diesem Zweck zur Verfügung gestellt.

\section{Förderpreis „Spezifische Immuntherapie“}

Der Förderpreis "Spezifische Immuntherapie“ der DGAKI, gestiftet von Allergopharma GmbH \& Co. KG, Reinbek, wird für eine herausragende Originalarbeit verliehen. Der Preis ist ausschließlich zur Auszeichnung besonderer wissenschaftlicher Arbeiten auf dem Gebiet der Allergologie und klinischen Immunologie bestimmt und dient der Förderung jüngerer Wissenschaftler - das Fördergeld muss projektbezogen verwendet werden. Für eine Prämierung kommen Arbeiten in Frage, die sich mit diagnostischen und therapeutischen Fragestellungen auf dem Gebiet der spezifischen Immuntherapie befassen. 2017 werden $5.000 €$ zu diesem Zweck zur Verfügung gestellt.

\section{Nachwuchsförderpreis der DGAKI}

Der Nachwuchsförderpreis der DGAKI, gestiftet von Siemens Healthcare Diagnostics $\mathrm{GmbH}$, Eschborn, wird jährlich für eine herausragende Originalarbeit verliehen. Der Preis ist ausschließlich zur Auszeichnung einer besonderen wissenschaftlichen
Arbeit auf dem Gebiet der Allergologie und klinischen Immunologie bestimmt und dient der Förderung jüngerer Wissenschaftler. Zurzeit werden jährlich bis zu $5.000 €$ zu diesem Zweck zur Verfügung gestellt. Für eine Prämierung kommen Arbeiten in Frage, die sich mit diagnostischen und therapeutischen Fragestellungen oder der Grundlagenforschung auf den Gebieten der Allergologie und klinischen Immunologie sowie verwandten Gebiete befassen.

\section{Für alle DGAKI-Preise gilt:}

Sie werden auf dem 12. Deutschen Allergiekongress, der vom 5. bis 7. Oktober in Wiesbaden stattfindet, verliehen. Der Preis ist für Wissenschaftler aus dem deutschen Sprachraum vorgesehen, die sich noch nicht in Lebensstellung befinden und die das 40. Lebensjahr zum Zeitpunkt der Annahme der Arbeit noch nicht vollendet haben. Ausgeschlossen sind Angestellte von Industriefirmen. Die Bewerber reichen bis zum 31. August 2017 eine Originalarbeit, die bis zu diesem Zeitpunkt publiziert oder zur Publikation angenommen ist, in deutscher oder englischer Sprache ein. Die Arbeit soll in elektronischer Form vorgelegt werden. Eine Verblindung ist nicht erforderlich. Der Erstautor hat handschriftlich zu versichern, dass er die Arbeit selbstständig erstellt hat. Die Jury kann bei mehr als einer eingereichten preiswürdigen Arbeit den Preis teilen. Je Arbeitsgruppe kann nur eine Arbeit eingereicht werden. Ein tabellarischer Lebenslauf ist ebenfalls einzureichen. Alle Unterlagen sind als PDF-Dateien per E-Mail an die Geschäftsstelle der DGAKI zu schicken.

Geschäftsstelle der DGAKI

Tel.: (0 30) 280471 00, E-Mail: info@dgaki.de

\section{Klaus-Kalveram-Preis}

Der 1995 gestiftete Klaus-Kalveram-Preis der Deutschen Stiftung Allergie ist nach dem Münsteraner Chemiker Dr. Klaus Kalveram (1943-1995) benannt und wird im Abstand von zwei Jahren in Zusammenarbeit mit dem Ärzteverband Deutscher Allergologen (AeDA) verliehen. Dem Stiftungsgedanken entsprechend dient er der Förderung von Nachwuchswissenschaftlern in der Allergologie. Der Preis ist mit $\mathbf{2 . 5 0 0} €$ dotiert.

Eingereicht werden können Arbeiten, die sich mit dem Themengebiet Allergologie wissenschaftlich beschäftigen und in sich abgeschlossen sind. Sie können aber auch bereits publiziert, sollten dann aber nicht älter als zwei Jahre sein.

Bewerber werden gebeten, ihre Arbeiten in deutscher oder englischer Sprache bis zum 15. August 2017 beim Vorsitzenden der Deutschen Stiftung Allergie in dreifacher Ausfertigung und zusätzlich elektronisch (an info@aeda.de) einzureichen. Eventuelle Anfragen sind ebenfalls an diese Adresse zu richten.

Die Arbeit muss ebenso wie das beigefügte Autoreferat ohne Namensvermerk oder Hinweis auf den Autor sein. Name und Anschrift des Bewerbers sind in einem geschlossenen Umschlag dem Manuskript beizufügen. Die Verleihung des Preises erfolgt anlässlich des 12. Deutschen Allergiekongresses, der vom 5. bis 7. Oktober 2017 in Wiesbaden stattfindet.

\section{AeDA-Geschäftsstelle}

Deutsche Stiftung Allergie,

Prof. Dr. Wolfgang Wehrmann

Blumenstraße 14, 63303 Dreieich

Tel.: (0 61 03) 63657

E-Mail: info@aeda.de

\section{Zwei weitere AeDA-Preise}

Der Ärzteverband Deutscher Allergologen (AeDA) prämiert den besten freien Vortrag auf dem 12. Deutschen Allergiekongress mit einem Preisgeld in Höhe von $750 €$.

Darüber hinaus prämiert der AeDA das beste klinisch relevante Poster des 12. Deutschen Allergiekongresses - ebenfalls mit einem Preisgeld in Höhe von $750 €$. 